ISBN 978-93-84422-79-0

6th International Conference on Humanities, Interdisciplinary Studies, Hospitality and Tourism

Management (HISHTM-17)

Singapore Aug. 10-11, 2017

\title{
An Investigation into a Computer-Aided Intimate Friendship Between Filipino Students and their Foreign Friends: Towards an Emerging Cross-Cultural Communication
}

\author{
Edgar R. Eslit, Ph.D. \\ St. Michael's College, Iligan City, Philippines
}

\begin{abstract}
Modern technology has enriched people's perspectives on how we communicate and establish intimate online friendship across cultural boundaries. With the emergence of Computer-aided Communication $(C A C)$, new concepts towards understanding the meaning of relationship maintenance, interdependence, commitment and relationship barriers in Cross-cultural communication situation have emerged. Others may find the use of modern communication technology dynamic while others find it artificial. Because of this, issues and concerns relating to the parameters of its use and how it affects friendship in the context of cross-cultural communication are opening all kinds challenging ideas for researchers all over the globe to explore. Hence, this study ventured into investigating the Computer-aided intimate friendship between Filipino students and their foreign friends: Towards an emerging Cross-cultural communication. Cultural Dimension, Relational Maintenance, and CAC theories served as primary theoretical backbones for this research. As this research was qualitative in nature, methodologies used include in-depth interviews, questionnaire and focus group discussion (FGD) which was voluntarily participated in by Filipino college students and their intimate online foreign friends. Results showed that informants utilized practical relationship maintenance strategies. Relative to publicized relationship maintenance strategies, however, another three notable strategies came to the surface. These are Material and financial support, Cyber-sensual activity and Constant and regular communication through CAC. Such strategies profoundly created strong interdependence and commitment that help broke certain relationship barriers between the collectivist and individualist informants. Further, informants convincingly explicated seven (7) online interaction practices that magnified the conceptualization of an emerging cross-cultural communication pattern. These practices are: a) Openness in communication; b) Willingness to commit to one's online friend; c) Benefiting from distance interaction; d) Strong Interdependence; e) Practical relationship maintenance strategies amidst relationship barriers; f) Active participation of social networks; and, g) Mutual support. Overall, the study brought the "techno-dyadic cross-cultural communication pattern" and "Computer-aided cross-cultural communication theory" to the fore.
\end{abstract}

Keywords: Culture, Language, Computer-aided, Cross-cultural communication, Intimate online friendship

\section{Introduction}

Study about intimate online friendship holds the promise of extending previous online relationship maintenance literature and adding new insights into the role of CAC in people's social lives. Learning how relationships are formed and maintained using the CAC can bring into focus the unspoken assumptions and biases of a face-to-face or physical relationship and communication practices. The study of Toyer (2011) in particular, pointed out that romances and dating cite left a gap in our understanding about emotional exchanges between online friends over the internet. Luft (2007) advances the field of research on internet friendships in that it investigates both platonic friendships and romances, and considers a variety of friendship sources. Further, Walther (2003) argues that research on relationship formation and relationship maintenance via CAC will reform not only the study of contemporary relationship dynamics, but help extend understanding of CAC across variety of domains. The lack of information about this area of communication interaction is creating a gap in the study 
of cross-cultural communication and the use of CAC. Thus, this dissertation investigated the issue about relationship maintenance strategies, commitment, interdependence, relational barriers and CAC which the target Filipino students utilized to maintain their relationship with their intimate online foreign friends. Overall, this study is hoped to yield empirical results that will help explain how CAC changes, redefines, or even alters people's traditional concepts about cross-cultural communication, CAC being known to quite possibly influence those with different cultural backgrounds who engaged in intimate online friendship relationship.

\section{Theoretical Framework}

Investigating for relationship maintenance, interdependence, commitment, relationship barriers and the use of CAC between Filipino students and their intimate online friends necessitates the presentation of pertinent theories that are relevant to the study. As the study is Cross-cultural in nature, this part of the paper presents the theories that would explicate the research problems.

Paramount to this is Hofstede's (1980) Cultural Dimension theory which stipulated the concepts of "collectivism" and "individualism". While collectivism and individualism can be studies in any culture, much of the research so far have been conducted on European and other Western cultures. A lot of researchers have found that Western people tend to be more individualist while East Asian people tend to be more collectivist. However, it is important to remember that many factors can influence this situation, so individual people within a particular culture can also differ in their levels of personal interdependence and views in life.. Another significant theory is the Relational Maintenance Theory. As cited in Canary and Stafford's (1994) study, relationship maintenance is defined as the actions and activities used to sustain a desired relationship situations. The theory elucidates that maintenance is required in order to keep a relationship working effectively, and that contentment will fall apart without the continuous adjustments to the so called maintenance strategies. Finally, Computer-aided Communication (CAC). As such, it has become one of the most common and widely used communication theories, even though it is fairly new. This theory is also known as Social Information Processing theory (Walther, 2011). Email, chat rooms, instant messaging, video chat, skype and the newest platforms of social media (Twitter, Facebook, LinkedIn, etc.) are some popular examples of CAC. Scholars asserted the ways that this kind of platform can shape communication and social interaction. For example, people can create the best relationships through social media in order to create certain understanding about them (Walther, 2011).

\section{Conceptual Framework}

Cross-cultural communication, as an existing field of study, endeavours to know how languages other than one's own can serve not only to help one understand what humans have in common, but also assist in the understanding of the diversity which underlines people's languages methods of constructing and organizing information. It is not a surprise then when one would attest that communicating across cultural borders is challenging. Because of this, research on cross-cultural communication often concentrates on accepting how individual variances affect one's capacity to interconnect with each other. In as much as most people are born and will grow with predetermined cultural background, having to interact with other people with different cultural bearings could represent a challenge. In a cross-cultural communication study, therefore, its core is to look at how people from diverse cultural backgrounds communicate in similar and different ways among themselves, and how they endeavor to communicate across cultures. Most studies, however, are based on physical or actual face-to-face communication. By and large, cross-cultural communication literatures by Gudykunst (1989), Hall (1959), Ting-Toomey (1984), Hofstede (1973) etc. are testament to this. Here, it would be worth mentioning, that as the world changes, the concept of cross-cultural communication also changes. The emergence of modern technology had brought instantaneous breakthroughs in terms of communication. The Internet and computers basically altered and improved the way people interact and communicate physically or even virtually across cultural boundaries. Hence, the inclusion of Hofstede's (1980) Cultural Dimension theory, 
Canary and Stafford's (1994) Relational Maintenance, Thibaut, J. W., \& Kelley, H . (1983). Interdependence and Commitment theory, and Walther's (2011) CAC theory were deemed essential for the paper's framework.

Due to limited literature, empirical studies on intimate online friendship using CAC hardly differentiate friendship from other forms of friendships, wherefore, remains attractive among language researchers. Studies should investigate the effects of online friendship among individuals in an intimate online friendship by looking into the confounding factors affecting cross-cultural communication. The schematic diagram that follows shows the different variables of the study.

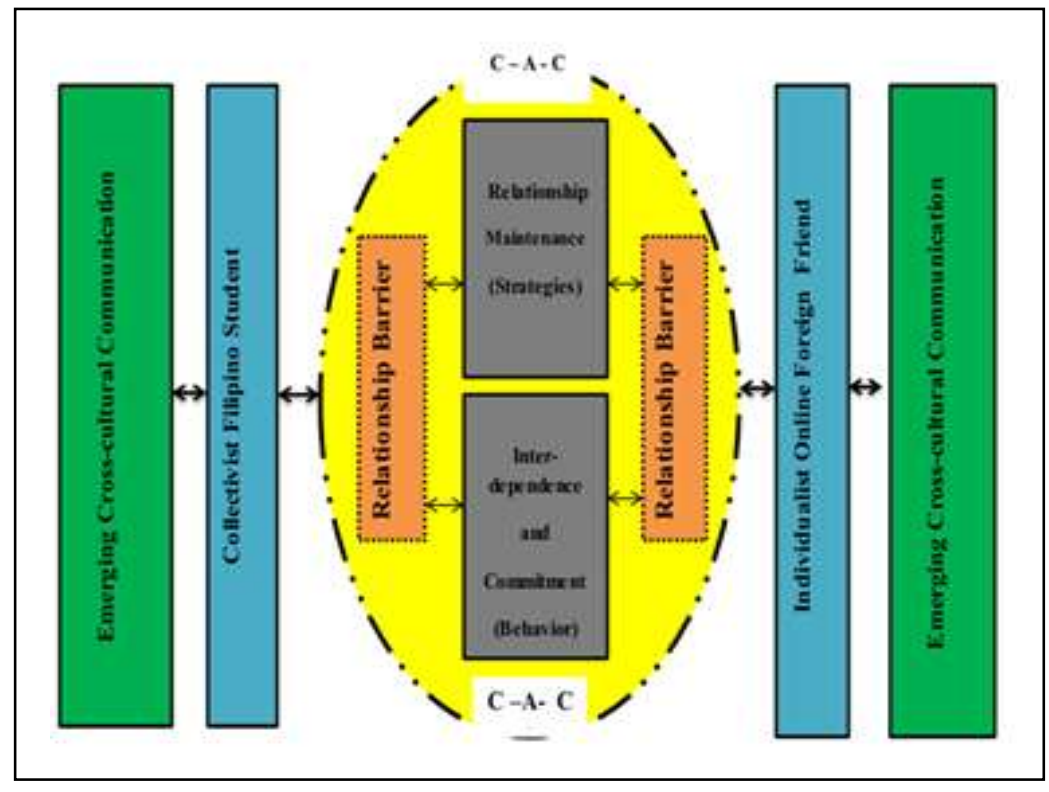

Fig 1: Schematic Diagram of the Study

\section{Statement of the Problem}

The advent of technology and the consequent prevalence of CAC have increased the chances of Internet users who have different beliefs, languages, values, and skills to be in contact with one another across the globe. Particular relationship maintenance strategies and online intimate friendship experiences are expected to emerge. However, current knowledge on how people with different cultural backgrounds develop interpersonal relationships with one another using CAC remains to be limited. There is clearly a need to extend understanding about the CAC and its use in the increasingly multicultural societies. Given the study's emphasis on relationship maintenance, interdependence, commitment, relationship barriers and the use of CAC, the following research questions were therefore proposed:

1) What is the profile of the target Filipino students and their intimate online friends in terms of age, gender, religion, nationality, language, educational status, and online activity duration?

2) What online relationship maintenance strategies are used by the informants to sustain their intimate online friendship?

a. Which of these relationship maintenance strategies suggest qualities of a collectivist society?

b. Which of these relationship maintenance strategies suggest qualities of an individualist society?

3) What shared interdependence behaviors do Filipino students have with their intimate online foreign friends?

3. a. Which of these reflect a collectivist society?

3. b. Which of these reflect an individualist society?

4) What kind of commitment do Filipino students have with their intimate online foreign friends? 
5) What barriers affect the relationship between the Filipino students and their intimate online foreign friends and how do they go about these?

6) How does Computer-aided Communication (CAC) practice affect relational maintenance and interdependence between the Filipino students and their foreign friends?

7) What cross-cultural communication pattern is emerging between the Filipino students and their intimate online foreign friends?

\section{Methodology}

This research is qualitative in nature. The conduct of the study relied so much on the combination of interview, observation, questionnaire and Focus Group Discussion (FGD). This entails both understanding the specific background context of a research setting and also spending a lot of time with the informants. In other words, in order to meet the standards of qualitative output, the researcher stood firmly on the ground where the study was conducted. Malinowski was the person who attributed to bringing out this kind of idea. He argued that to truly understand people, their language, interaction, culture and even their relationship, one has to spend ample time to be with the informants to learn not only their language and situation but their feelings, emotions and relationship as well (Malinowski, 1967). Becker (2007) further argued about the epistemological aims of quantitative research that it is not fundamentally different from qualitative research - it is just that the standards, questions, and methods used tend to be diverse. Becker distinctly labelled this idea in terms of breadth, precision, and accuracy; hence, the same precision was epitomized and applied in this study.

The profile and demographic information of the informants were gathered for the purpose of describing the informants. As stipulated early on, one of the main sources of data gathering was an in-depth interview, which was recorded every session. In the study of McCracken (1988), he stipulated that the interview will allow the researcher to get into the mind of participants being interviewed and will experience their world as they see it. Further, in-depth interviews were used as they are excellent means of grasping the meanings attached to relationship maintenance strategies, interdependence, and commitment in a relationship (Arditti and Prouty, 1999). Closed-ended questions regarding age, gender, religion, nationality, language, educational status, and online activities among the informants were asked. The in-depth interviews utilized a set of open-ended questions and probes designed to explore the meanings individual respondents attached to their words, how they saw their relationship and distance as well as how informants evaluated their partner and the strengths of their relationship. In so doing, relational maintenance, relational barriers, interdependence, commitment and the use of CAC were thoroughly scrutinized.

\section{Data Collection}

A great deal of recorded interviews and journal notes were generated as a result of the in-depth interviews and, given the exploratory nature of the paper, the study was focused on the experience of both partners. Persuasive and exhaustive efforts were exerted in gathering the data in order to corroborate the information given by both intimate online friends and to fully understand the online experiences of both parties. It is not uncommon to study only one person in the dyad to learn about intimate relationships of both partners (Rusbult, 1983), but this study seemed to succeed in doing the extreme. Instead of just focusing on only one personal account about intimate online friendship experiences, this study interviewed both parties in an intimate online relationship.

Based on the profile of the informants, the youngest was eighteen (18) years old and the oldest was thirty (30) years old, giving an average age of 21 years old. Smelser and Erikson (1980) explained that a societal expectation generalizes for individuals to begin early adulthood at approximately the age of 18 to underscore the legal or psychological points such as that of being in the "age of reason". This age group was selected, as opposed to younger respondents, due to their presumed greater maturity and independence (Smelser and Erikson, 1980). In the Philippine context, younger students are more likely to have a higher level of dependence on their 
parents in terms of emotional, financial and personal responsibilities including their academic obligations. Hence, the participants included were at least 18-year old for both SIs and FIs. The open-ended nature of the interview allowed better exploration of the topic and in finding out how their online attachment to their intimate foreign friends influenced their online relationship maintenance strategies, interdependence and commitment with each other.

\section{Results}

Consistent with the qualitative research approach, presenting the prevailing output relative to the objectives of the current study is the main goal. The study basically aimed at finding out how the informants who belong to the collectivist and individualist communities dealt with issues concerning their relationship maintenance, interdependence, commitment, relational barriers and the use of CAC in their regular day-to-day communication or interaction. The questions were fully answered by the SMC student informants (SIs) and their intimate online foreign friends otherwise known as the foreign informants (FIs). One of the concentrations of the study involved exploring the ways in which intimate online friends stayed connected and dealt with any difficulty posed by their physical distance from each other. Dense data of narratives and the ensuing analyses attained through in-depth interviews and focus group discussion (FGD) showed significant relationship maintenance strategies, interdependence, commitment, and communication patterns that were not previously discussed in other literature on intimate online friendship. These brought emerging insights about Cross-cultural communication pattern.

Based on the data collected, ten (10) prevalent online interaction practices came to the surface as the result of the constant interaction between the Filipino students and their intimate online friends or also known as SIs and FIs informants. With these ten (10) online interaction practices, they served as potent knitting glue in forming the emerging cross-cultural communication pattern. These online interaction practices are: a) Openness in communication; b) Willingness to commit online; c) Benefiting from distance interaction; d) Strong Interdependence; e) Practical relationship maintenance strategies amidst relationship barriers; f) Active participation of social networks; g) Mutual support; h) Material and financial support; i) Cyber-sensual activity; and, j) Constant and regular communication through CAC. Combining the ten (10) online interaction practices, with CAC, the researcher postulated the idea of calling the emerging communication pattern as "techno-dyadic cross-cultural communication pattern" for it occurred between two individuals who belong to two different cultural backgrounds and was happening in an online "dyadic" communication situation. Using CAC, informants of the study made use of them to initiate the necessary "interaction" that made both parties acquire personal information until such time that they were able to establish their intimate friendship online. All these interaction practices were made possible with the use of CAC in "dyadic" context. As such, it can be further illustrated using this formula: "techno-dyadic initiation + techno-dyadic acquisition $=$ techno-dyadic crosscultural communication". As this has created a significant communication pattern, consideration about the ten (10) online interaction practices needs serious attention.

Generally speaking, the informants expressed complete trust with their intimate online friends, citing it as making their online friendship successful. Nonetheless, added effort must be made to keep their interaction with their online friend always available since distance affects their communication activities. Informants identified no major problems with their online intimate friends because of CAC, who, also signified that they, though belonging to different cultural backgrounds, felt their friendship are always getting stronger. With the absence of the actual physical interaction, the use CAC made their virtual interaction possible. If and when actual physical interaction fails, it can be supplemented or even intensified with the use of CAC's synchronous and asynchronous channels (Walther, 2011). This, therefore, advances the idea of an online "dyad". Such idea is supportive of the traditional dyadic communication (see Osgood, Schramm and Berlo's dyadic communication models), but with the emergence of the modern communication technology, such idea has been modified, for, instead of using the traditional physical and face-to-face interaction to complete the concept of a "dyad", modern communication technology has made its own way in making a particular online interaction possible. Hence, the 
current communication pattern, as amplified by the ten (10) online interaction practices will help us understand the intricacies that allow the building of an emerging cross-cultural communication pattern.

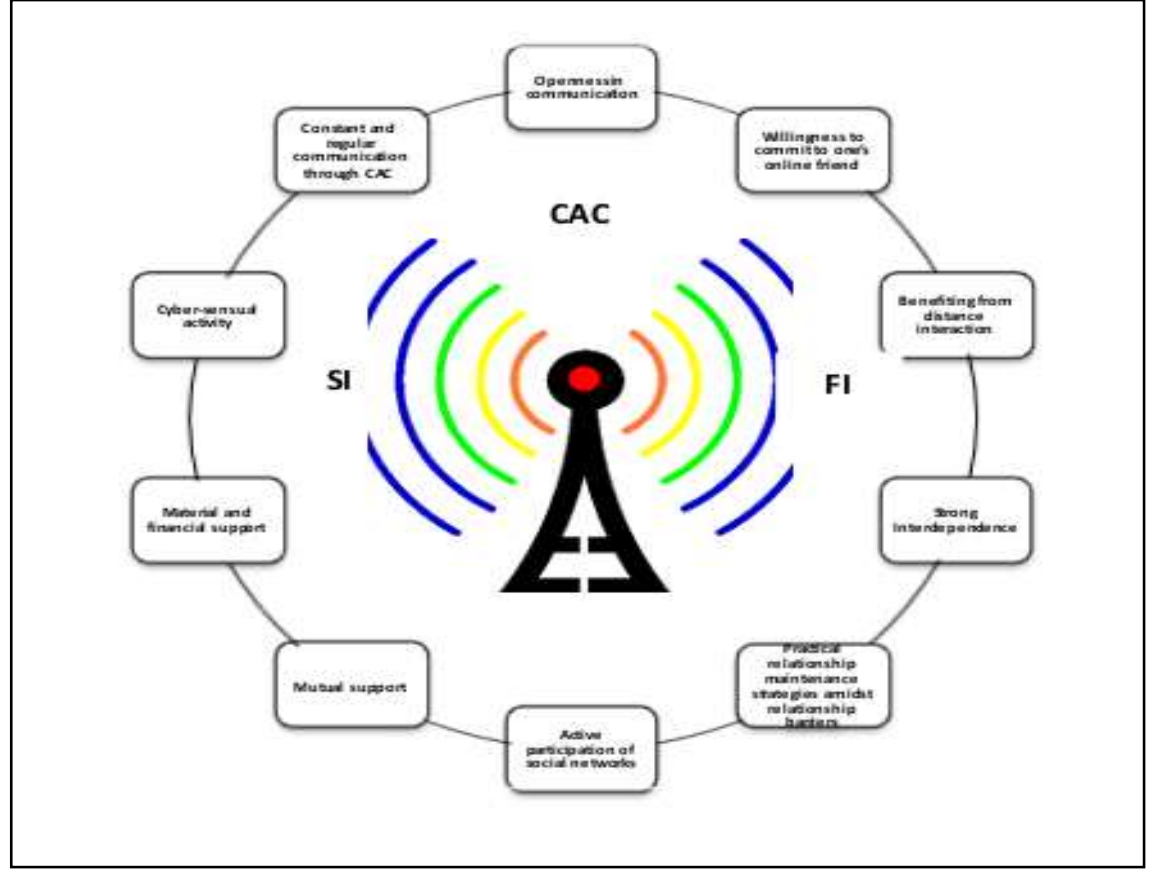

Fig. 2: The SIs - FIs Interaction Process leading to an Emerging Techno-Dyadic Cross-Cultural Communication Pattern

\section{Conclusion}

The current in-depth examination of the issue allowed better insights and significant merits to emerge. Online friendship is very fascinating and rewarding for both online friends (SI and FI). The mere fact that distance necessitated extra effort to communicate, for the informants it helped develop their practical relationship maintenance, commitment, interdependence and in breaking certain forms of relationship barriers. To date, five months after the conduct of this study, a pair of informants has tied the knot in the sacrament of matrimony. This development, in effect, gave a new and better perspective towards online friendship in the context of cross-cultural communication. Cultural dimension, relationship maintenance, and CAC theories, with which this study was anchored, were magnified in this study. Though such theories can be assumed as based on the face-to-face or physical social interaction, their magnification is made possible with the use of CAC. Not to negate the beauty of the longstanding concept of the face-to-face or physical communication interaction, but the ever-increasing access to Internet, indeed, greatly expanded people's language and social capacity to form a firm intimate online friendship. As an interactive medium, CAC allowed the respondents, whether collectivists or individualists, to overcome great distances in order to communicate with their intimate partners almost instantaneously according to Bargh and McKenna (2004). Computer-aided Communication (CAC), in a variety of forms, had become integral to the initiation, development, and maintenance of interpersonal relationships (Eslit, 2013). Indeed, across cultural boundaries, CAC is involved in the subtle shaping of communication in every communicative and relational context possible that bring forth the birth of techno-dyadic cross-cultural communication pattern and Computer-aided Cross-cultural Communication Theory to the fore.

\section{Theory Arising from the Study}

Having completed this study, one grounded theory was formulated by the researcher to explain the reasons behind the success of the intimate online friendship between the Filipino students (collectivists) and their foreign online friends (individualists). The theory is proposed to be called "Computer-aided Cross-cultural Communication" theory. The Computer-aided Cross-cultural Communication Theory. As defined early on, Cross-cultural communication as a field of study that looks at how people from different cultural backgrounds 
communicate, in similar and different ways among themselves, and how they endeavor to communicate across cultures (Gudykunst, 2002). In relation to Computer-aided Cross-cultural communication theory, the researcher underscored the statement that says "people from different cultural backgrounds communicate, in similar and different ways among themselves, and how they endeavor to communicate across cultures". Indeed, the current theory amplified the words "different ways" as it connotes practicality, sensibility and efficiency. Why practical, sensible and efficient? Given the concept of internationalization where people are now living in one global village and where technology is always connected in almost everything people do, it can be assumed that people will be left behind or expire in isolation if they will not accept the benefits provided by modern communication technology. Why cogitate distance as reason for isolation when CAC can make the necessary connection? Why stay alone when technology can provide better companion? And, why limit interaction with just one person or a segment of the society when there is an enormous opportunity to reach out to different kinds of people around the globe in just one click of the mouse? Indeed, CAC can facilitate the indispensable interaction while providing all kinds of methods available for communication.

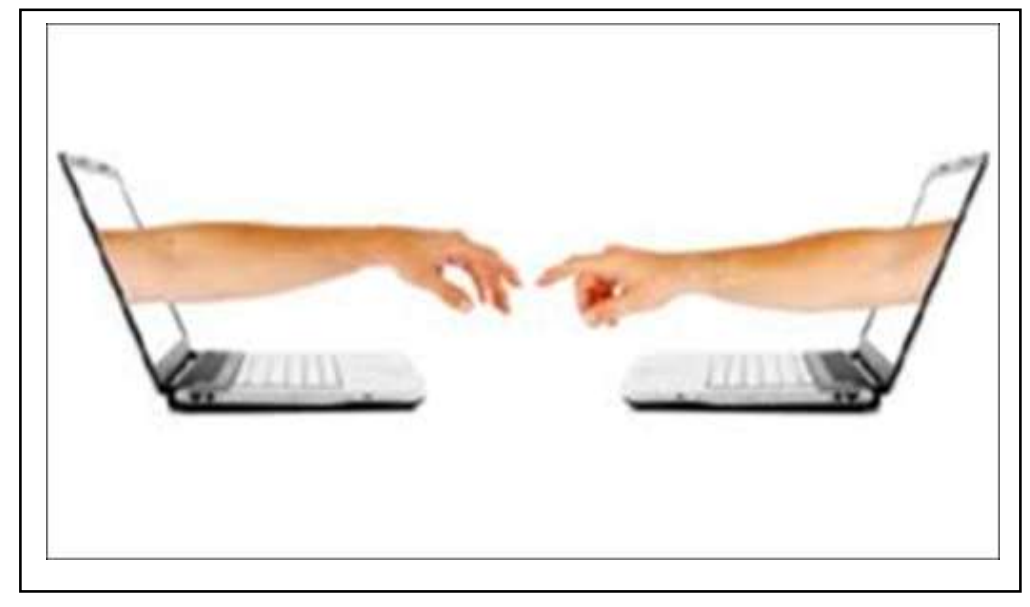

Fig. 3: Genesis of Computer-aided Cross-cultural Communication Theory

\section{Recommendations for Future Research}

Since CAC has proven to pave the way in establishing, sustaining and enhancing online relationship maintenance, interdependence, commitment and in breaking certain form of relational barriers across cultural boundaries, it would be of essence if other researchers will venture into studying local male and female informants, who engaged in an intimate online friendship using the CAC with other foreign nationals. Second, it would also be significant to make a quantitative and comparative study among college students from private and public colleges or universities who venture into this kind of activity to see the veracity of their relationship. Lastly, a longitudinal study will contribute important literature about intimate online friendship in order to understand how it affects relationship formation and cohabitation. With the present study's restricted number of informants, results may not be adequate to make conclusive generalizations about the steadfastness of CAC in maintaining a strong intimate online friendship relationship. A similar study involving a wide variety of participants would strengthen the findings of the study.

\section{References}

[1] Arditti, J.A., \& Prouty, A. (1999). Change, disengagement, and renewal Relationship dynamics between young adults with divorced parents and their fathers. Journal of Marital and Family Therapy, 25, 61-81.A. Cichocki and R. Unbehaven, Neural Networks for Optimization and Signal Processing, 1st ed. Chichester, U.K.: Wiley, 1993, ch. 2, pp. 45-47. 
[2] Canary, D. J.; Stafford, L., \& Semic, A. (2002). A panel study of the associations between maintenance strategies and relational characteristics. Journal of Marriage and the Family, 64, 395-406.W. D. Doyle, "Magnetization reversal in films with biaxial anisotropy," in Proc. 1987 INTERMAG Conf., 1987, pp. 2-6.

[3] Eslit, E. (2014). Computer Assisted Language Teaching: Learning Without Dust. https://www.academia.edu/11536522/COMPUTER_ASSISTED_LANGUAGE_TEACHING_LEARNING_WITHO UT_DUST N. Kawasaki, "Parametric study of thermal and chemical nonequilibrium nozzle flow," M.S. thesis, Dept. Electron. Eng., Osaka Univ., Osaka, Japan, 1993.

[4] Gudykunst, W.B. (2002). Intercultural Communication Theories. In Gudykunst, W.B. and Mody B. (eds.) Handbook of International and Intercultural Communication (pp 183-205). London: Sage Publications.Transmission Systems for Communications, $3^{\text {rd }}$ ed., Western Electric Co., Winston-Salem, NC, 1985, pp. 44-60.

[5] Gudykunst, W.B. (2002). Intercultural Communication Theories. In Gudykunst, W.B. and Mody B. (eds.) Handbook of International and Intercultural Communication (pp 183-205). London: Sage Publications.

[6] Hofstede, G. "Dimensionalizing Cultures: The Hofstede Model in Context". ScholarWorks@GVSU. Online Readings in Psychology and Culture. Retrieved 6 September 2015)

[7] Luft. C. (2007). Meeting Online Friends: Personal Relationships in the 21st Century. ww.seriousleisure.net/ uploads/ 8/3/3/8/.../ luft.ma.meeting_internet_friends1.pdf

[8] Leake, D., \& Black, R. (2005). Essential tools: Cultural and linguistic diversity: Implications for transition personnel. Minneapolis, MN: University of Minnesota, Institute on Community Integration, National Center on Secondary Education and Transition.

[9] McCracken, G.D., (1988). The long interview. Newbury Park, CA: Sage.

[10] Smelser, N.J., \& Erikson, E.H. (1980). Themes of work and love in adulthood. Cambridge, MA: Harvard University Press.

[11] Thibaut, J. W., \& Kelley, H . (1983) . The social psychology Newlbrk : Wiley.

[12] Toyer, R. L. (2011), "Factors of adoption: Initiating relationships using online dating sites" (2011). UNLV Theses/Dissertations/ Professional Pap ers/Capstones. Paper 1003. Walther (2003

[13] Walther, J. (2011). Theories of computer-mediated communication and interpersonal relations. In M. L. Knapp \& J. A. Daly (Eds.), The Sage handbook of interpersonal communication (4th ed., pp. 443-479). Thousand Oaks, CA: Sage. 